\title{
Reply to: "QT Prolongation With Hydroxychloroquine and Azithromycin For The Treatment of COVID-19: The Need For Pharmacogenetic Insights"
}

\author{
James Hummel ${ }^{1}$ and joseph akar ${ }^{1}$ \\ ${ }^{1}$ Yale University School of Medicine
}

August 17, 2020

We thank Medina et al. for their interest in our recent work on QTc prolongation associated with treatment of COVID-19 patients with hydroxychloroquine and azithromycin. As they appropriately point out in their letter, genetic variation is likely a significant determinant of QT prolongation in the population at large and in COVID-19 patients specifically. While drugs causing acquired long QT syndrome and torsades de pointes are generally blockers of $\mathrm{I}_{\mathrm{Kr}}$, repolarization results from the aggregate of multiple inward and outward currents. Patients with sub-clinical defects in any of these ion channels can have normal or only slightly prolonged baseline QT intervals, but may possess decreased repolarization reserve leading to an exaggerated response to $\mathrm{I}_{\mathrm{Kr}}$ blockade (1). In our study, a baseline QTc of $>460 \mathrm{~ms}$ was associated with excessive QTc prolongation, and this likely represents a group of patients with sub-clinical cardiac ion channel mutations (so called "first hit") (2). We also agree that many patients with latent mutations demonstrate a normal baseline QT, which gets prolonged with the addition of a drug or a change in the clinical condition "second hit" (3). The patients in our study who exhibited QTc prolongation were generally acutely ill, and displayed "multiple hits" that led to QTc prolongation and it is certainly plausible that many may have had sub-clinical cardiac ion mutations. We therefore wholeheartedly agree that pharmacogenetics should be considered in studies of drug-induced QT prolongation, however this information is rarely available to include for acutely ill patients. And while it makes sense to obtain genetic profiles prior to administration of QT-prolonging medications, that can only be performed in the elective outpatient setting, while taking into consideration medical, ethical and social issues related to asymptomatic genetic screening (e.g. cost, reimbursement, informed consent, etc...). There is significant interest in building genomic databases, and when this becomes a reality for the population at large we believe that genetic information should certainly be included in studies of QT prolongation.

1. Roden DM Long QT syndrome: reduced repolarization reserve and the genetic link. J Intern Med. 2006 Jan; 259(1):59-69.

2. Napolitano C, Schwartz PJ, Brown AM, et al. Evidence for a cardiac ion channel mutation underlying drug-induced QT prolongation and life-threatening arrhythmias. J Cardiovasc Electrophysiol. 2000;11:691-6

3. Sauer AJ and Newton-Cheh C. Clinical and genetic determinants of torsade de pointes risk. Circulation. 2012;125:1684-94. 